\title{
When the Immunoreactions Don't Help.
}

\author{
W.T. Gunning
}

University of Toledo, Department of Pathology, Toledo, USA

The practice of pathology has undergone numerous "eras" since the time of Rudolf Carl Virchow (18211902), considered the father of modern pathology, who published a book entitled Cellular Pathology in 1858 that has left an indelible footprint for much of the discipline of studying/diagnosing disease as we know it today ${ }^{1}$. Virchow advanced cell theory as proposed by earlier scientist, that all living organisms are composed of one or more cells, that the basic unit of an organism is the cell, and specifically, he stressed that all cells come from cells ${ }^{1}$. He described many disease processes and states and the names of numerous conditions, diseases and physiologies are commonly known to non-physicians including leukemia, embolism, and thrombosis to cite just a few.

The "eras" of modern pathology since the mid eighteen hundreds include the utilization of basic special light microscopic stains and dyes procedures, the advent and utilization of histochemical techniques in the early to mid-1950's (still an essential technique to evaluate muscle disease), the development and application of the electron microscope during the 1950s-1980's, and the development of antibody and labeling techniques in the 1980s with refinement that continues today. Most recently, applications of our knowledge of genomics has led to an expanding field of pathology: molecular pathology that holds great promise for more precise diagnosis of disease with goals of developing personalized medicine in the future.

With the advent of immunocytochemistry, known to most as immunohistochemistry, most electron microscope laboratories have closed with the exception of most academic training programs and reference labs. This has happened for a variety of reasons but mostly due to financial considerations as ultrastructural pathology requires expensive microscopes, ancillary equipment and knowledgeable technical staff. The application of antibody labels can be readily performed by skilled histotechnicians without any specialized equipment; current applications can now be easily performed with automated immuno-"stainers". A compounding factor includes declining educational opportunities for pathologists in training; thus young pathologists tend to be intimidated by ultrastructural morphology, both normal and pathologic, as they are not being adequately introduced to the application of EM in pathology.

What happens if your light microscopic morphology and special stains identify a general disease process but not a specific diagnosis? What if 10-15 antibodies employed don't help? What if you do not have access to an ultrastructural facility and you have a difficult case to diagnose? Such cases are referred to outside experts and reference laboratories. Sometimes the experts "get it wrong"! To highlight such a dilemma, a recent case of ours that was misdiagnosed by light microscopy with numerous non-specific will be presented. Briefly, a circumscribed perirectal tumor in a 68 year old man was diagnosed as a gastrointestinal stromal tumor (GIST) at the time of frozen section; subsequently, the spindle cell neoplasm was diagnosed as a benign smooth muscle tumor (leiomyoma) when supporting immunoreactions were all found to be negative ${ }^{2,3}$. The diagnosis was based upon the bland morphology of the tumor and the statistical likely hood that the tumor would probably arise from smooth muscle ${ }^{4}$; thus the lesion was diagnosed as a spindle cell neoplasm.......until reviewed by electron microscopy! 
[1] Scultz, M. Rudolf Virchow, Emerg. Infect. Dis., 14(9): 480-81, 2008.

[2] Antonescu, C.R., et al. Dedifferentiation in Gastrointestinal Stromal Tumor to an Anaplastic KIT Negative Phenotype - a Diagnostic Pitfall. Morphologic and Molecular Characterization of 8 Cases Occurring either de-novo or after Imatinib Therapy. Am. J. Pathol. 37(3):385-392, 2013.

[3] Terada, T. Smooth muscles and stem cells of embryonic guts express KIT, PDGFRRA, CD34 and many other stem cell antigens: suggestion that GIST arise from smooth muscles and gut stem cells. Int. J. Clin. Exp. Pathol. 6(6):1038-45, 2013.

[4] Nakayama, T., et al. Expression of vascular endothelial growth factor and its receptors VEGFR-1 and 2 in gastrointestinal stromal tumors, leiomyomas and schwannomas. World J. Gastroenterol. 12(38):6182-87, 2006.

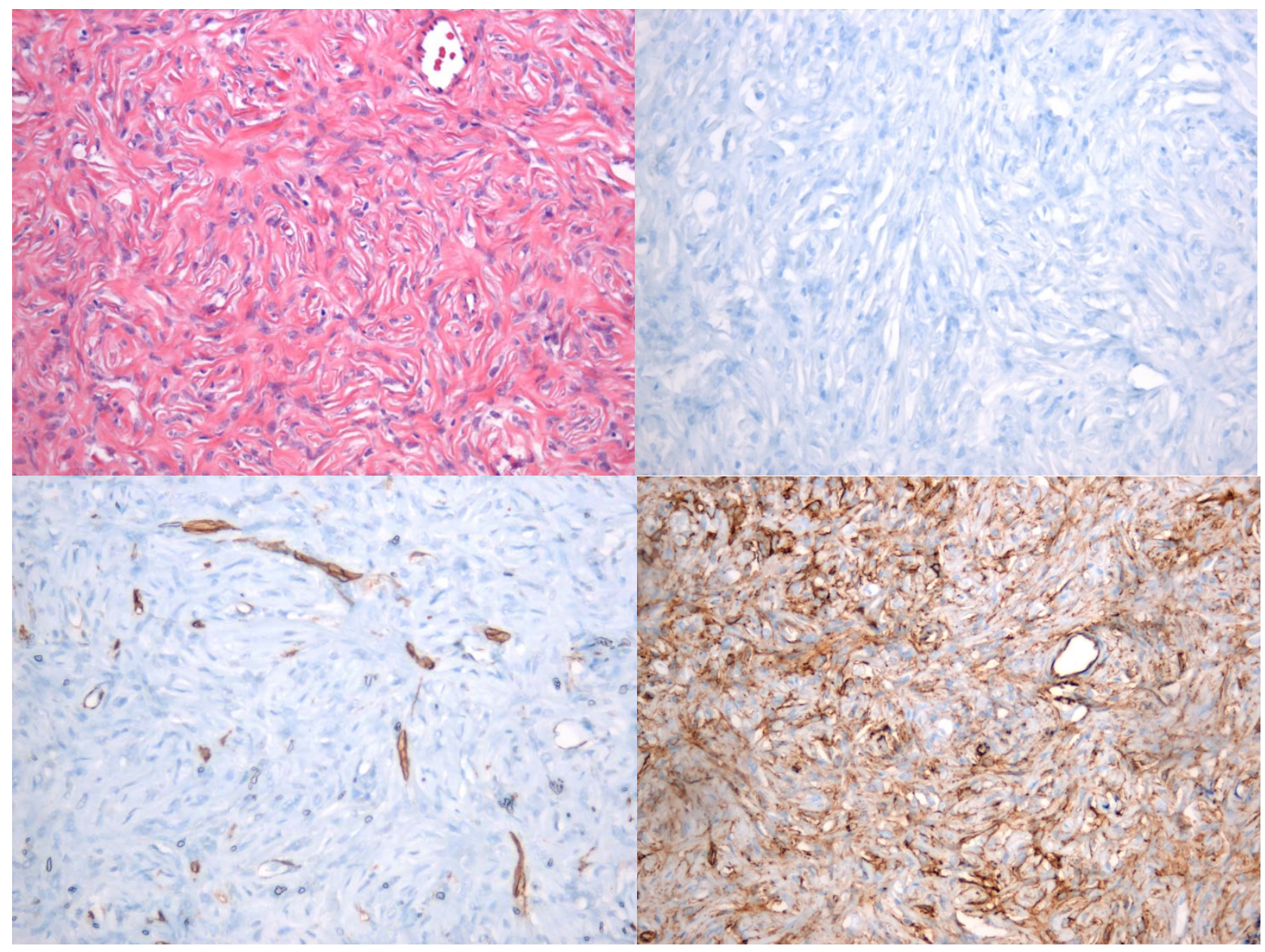

Figure 1. The H\&E stained tumor reveals whorls of spindle-shaped cells. 20X

Figure 2. CD117 antibody specific for GIST tumors was negative. 20X

Figure 3. Antibody specific for smooth muscle actin was only positive in vessles. 20X

Figure 4. CD34 antibody was membrane positive. 20X 